Supporting Information for

\title{
Synthesis of Pt-Ni Octahedra in Continuous-flow Droplet Reactors for the Scalable Production of Highly Active Catalysts toward Oxygen Reduction
}

Guangda Niu, ${ }^{\dagger}{ }^{\S}$ Ming Zhou, ${ }^{\dagger}$ Xuan Yang, Jinho Park, ${ }^{\dagger}$ Ning Lu, J Jinguo Wang," Moon J. Kim," Liduo Wang, ${ }^{\S}$ and Younan Xia ${ }^{*, \dagger}$,

${ }^{\dagger}$ The Wallace H. Coulter Department of Biomedical Engineering, Georgia Institute of Technology and Emory University, Atlanta, Georgia 30332, United States

${ }^{\S}$ Key Lab of Organic Optoelectronics and Molecular Engineering of Ministry of Education, Department of Chemistry, Tsinghua University, Beijing 100084, China

${ }^{\ddagger}$ School of Chemistry and Biochemistry, Georgia Institute of Technology, Atlanta, Georgia 30332, United States

"Department of Materials Science and Engineering, University of Texas at Dallas, Richardson, TX 75080, United States

*Corresponding author: younan.xia@bme.gatech.edu 


\section{Chemical and Materials}

Platinum(II) acetylacetonate (Pt(acac) $\left.)_{2}, 99.99 \%\right)$, nickel(II) acetylacetonate (Ni(acac) $\left.2,95 \%\right)$, oleylamine (OAm, 70\%), oleic acid (OAc, 90\%), benzyl ether (BE, 98\%), tungsten hexacarbonyl $\left(\mathrm{W}(\mathrm{CO})_{6}, 99.99 \%\right)$, and acetic acid $(\mathrm{HAc}, 99.5 \%)$ were all obtained from Sigma-Aldrich and used as received.

\section{Preparation of the Precursor Solution}

$20 \mathrm{mg}$ of $\mathrm{Pt}(\mathrm{acac})_{2}(0.051 \mathrm{mmol})$ and $8 \mathrm{mg}$ of $\mathrm{Ni}(\mathrm{acac})_{2}(0.031 \mathrm{mmol}), 2 \mathrm{~mL}$ of OAm, $1 \mathrm{~mL}$ of OAc were introduced into $7 \mathrm{~mL}$ of BE. To avoid the oxidation of nickel during a synthesis, the air was excluded from the mixture by bubbling with argon and then filling the vial with argon. The mixture was then heated to $70{ }^{\circ} \mathrm{C}$ for $10 \mathrm{~min}$ to dissolve all the solids. After cooling down to room temperature, $20 \mathrm{mg}$ of $\mathrm{W}(\mathrm{CO})_{6}$ was introduced. Under the protection of argon, the solution was heated to $70{ }^{\circ} \mathrm{C}$ again until all the $\mathrm{W}(\mathrm{CO})_{6}$ had been dissolved. Afterwards, the precursor solution was allowed to cool down to room temperature prior to introduction into the device.

\section{Synthesis of 9-nm Pt-Ni Octahedra in a Continuous Flow of Droplet Reactors}

The device was assembled from commercially available components, including PTFE tubes with an inner diameter of $1.58 \mathrm{~mm}$ (typically used at a length of $7.5 \mathrm{~m}$ ), a glass syringe, and a syringe pump as shown in Figure 1. During a standard synthesis, the fluidic system was operated at a flow rate of $0.15 \mathrm{~mL} \mathrm{~min}^{-1}$. The PTFE tube was immersed in an oil bath with its temperature set to $230{ }^{\circ} \mathrm{C}$. The residence time, determined by the flow rate and the length of the PTFE tube, was set to $30 \mathrm{~min}$. After reaction, the solution was collected in a centrifuge tube. After the mixture had cooled down to room temperature, the $\mathrm{Pt}-\mathrm{Ni}$ nanocrystals were precipitated out by sequentially adding toluene $(15 \mathrm{~mL})$ and ethanol $(15 \mathrm{~mL})$ into the as-obtained solution $(10 \mathrm{~mL})$. The supernatant was removed by centrifugation at 6,000 rpm for $10 \mathrm{~min}$. The resulting Pt-Ni octahedra could be easily dispersed in an organic solvent such as toluene or hexane. All samples of Pt-Ni octahedra reported in this paper were produced using fluidic devices fabricated from $1.58 \mathrm{~mm}$ PTFE tubes unless otherwise mentioned.

To further increase the throughput, we also explored the synthesis of Pt-Ni octahedra in a fluidic device assembled from PTFE tubes with an inner diameter of $3.00 \mathrm{~mm}$ (typically used at a length 
of $5.5 \mathrm{~m}$ ). In this case, the flow rate was also increased to $0.30 \mathrm{~mL} \mathrm{~min}{ }^{-1}$ to maintain a continuous flow. All other details and work-up process were the same as those used for the synthesis in PTFE tubes with an inner diameter of $1.58 \mathrm{~mm}$. A TEM image of the products is shown in Figure S3.

\section{Preparation of Pt-Ni/C Catalysts}

In a typical process, $10 \mathrm{~mL}$ of the suspension of the $\mathrm{Pt}-\mathrm{Ni}$ octahedra in toluene was mixed with Ketjen Black $(\mathrm{KB})$ carbon suspended in toluene under ultrasonic agitation for $3 \mathrm{~h}$ to achieve a $\mathrm{Pt}$ loading of $\sim 15 \mathrm{wt} \%$. The resulting Pt-Ni/C catalyst was centrifuged at $12,000 \mathrm{rpm}$ for $15 \mathrm{~min}$, and then added into $20 \mathrm{~mL}$ of acetic acid and heated at $60{ }^{\circ} \mathrm{C}$ for $2 \mathrm{~h}$. The final catalyst was washed six times with ethanol and dried for $30 \mathrm{~min}$ in an oven set at $70{ }^{\circ} \mathrm{C}$ prior to the ORR measurements.

\section{Characterizations}

\section{Morphological, structural, and elemental characterization}

Transmission electron microscopy (TEM) images were taken using a Hitachi HT7700 microscope operated at $120 \mathrm{kV}$. High-resolution TEM (HRTEM), high-angle annular dark-field scanning TEM (HAADF-STEM), and energy dispersive X-ray spectroscopy (EDS) mapping analyses were performed using a JEOL ARM200F microscope (JEOL, Tokyo, Japan) with STEM aberration corrector operated at $200 \mathrm{kV}$. The metal contents in the as-obtained catalysts were determined using inductively coupled plasma mass spectrometry (ICP-MS, Perkin-Elmer, NexION 300Q).

\section{Electrochemical measurements}

In a typical procedure, catalyst ink with a concentration of $1 \mathrm{mg} \mathrm{mL}^{-1}$ was first prepared by dispersing the $\mathrm{Pt}-\mathrm{Ni} / \mathrm{C}$ powder in a mixture of DI water, isopropanol alcohol (IPA), and 5\% Nafion $\left(\mathrm{V}_{\text {water }}: \mathrm{V}_{\text {IPA }}: \mathrm{V}_{5 \% \text { Nafion }}=0.5: 0.5: 0.02\right)$ under ultrasonication for $30 \mathrm{~min}$. The concentration of $\mathrm{Pt}$ in the final dispersion was determined by ICP-MS. Finally, a suspension containing $1.23 \mu \mathrm{g}$ Pt was deposited on a pre-cleaned glassy carbon electrode (Pine Research Instrumentation) and allowed to dry. For a commercial Pt/C catalyst (20 wt\% $3.2 \mathrm{~nm}$ nanoparticles on Vulcan XC-72 carbon support, Premetek Co.), the amount of Pt loading was $2.42 \mu \mathrm{g}$. A HydroFlex reference 
electrode (ET070, Edaq Inc.) and a Pt wire were used as the reference and counter electrodes, respectively. The potentials of the reference electrode were calibrated using a home-built reversible hydrogen electrode (RHE).

After loading, the working electrode was cleaned through a chronoamperometry method by setting the potential of the working electrode at $0.05 \mathrm{~V}$ for $60 \mathrm{~s}$. Then the electrode was cycled in a $\mathrm{N}_{2}$-saturated $0.1 \mathrm{M} \mathrm{HClO}_{4}$ (GFS Chemicals) solution for 100 cycles between 0.08 and $1.1 \mathrm{~V}$ at a rate of $100 \mathrm{mV} \mathrm{s}^{-1}$. The underpotential deposition (UPD) of $\mathrm{Cu}$ was conducted in a solution of $50 \mathrm{mM} \mathrm{H} \mathrm{SO}_{4}$ and $50 \mathrm{mM} \mathrm{CuSO}$. The electrochemical active surface area (ECSA) of the catalysts was measured through $\mathrm{Cu}_{\mathrm{UPD}}$, which is more appropriate than $\mathrm{H}_{\mathrm{UPD}}$ in the case of $\mathrm{Pt}-\mathrm{Ni}$ nanocrystals. The charge density for $\mathrm{Cu}_{\mathrm{UPD}}$ was assumed to be $480 \mu \mathrm{C} \mathrm{cm}^{-2}$. The oxygen reduction polarization curves were measured in an $\mathrm{O}_{2}$-saturated $0.1 \mathrm{M} \mathrm{HClO}_{4}$ solution at a scan rate of $10 \mathrm{mV} \mathrm{s}^{-1}$ and a rotation speed of 1,600 rpm. The kinetic current density $j_{k}$ at $0.9 \mathrm{~V}$ was derived from the Koutecky-Levich equation:

$$
\frac{1}{j}=\frac{1}{j_{k}}+\frac{1}{B \omega^{1 / 2}}
$$

Where $j$ is the measured current density, $B$ is a constant, and $\omega$ is the rotation speed. The electrochemical measurements were performed using a $\mathrm{CHI} 600 \mathrm{E}$ potentiostat $(\mathrm{CH}$ Instrument, Austin, TX). The kinetic currents were corrected by iR-compensation. 


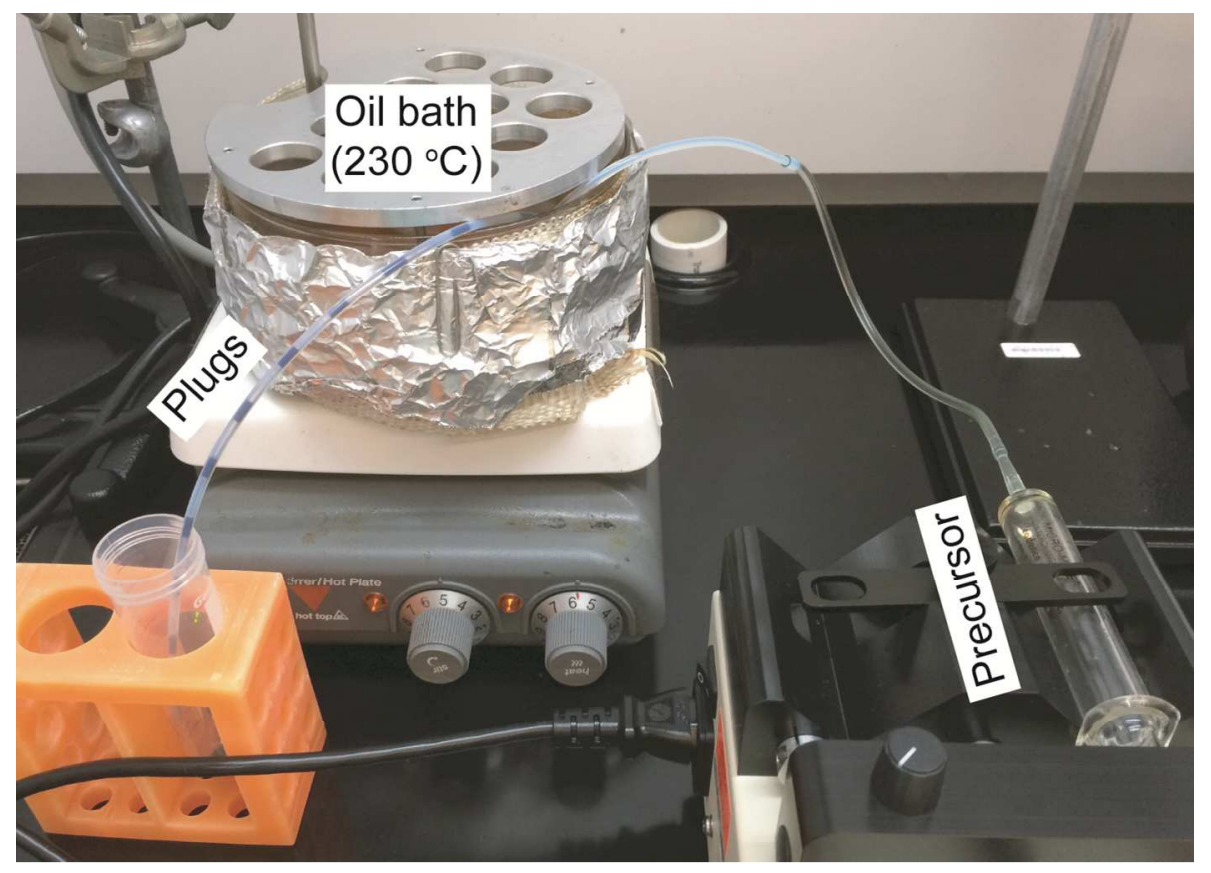

Figure S1. Photograph showing the formation of plugs in the PTFE tube as the solution entered the reaction zone when $\mathrm{W}(\mathrm{CO})_{6}$ was used at a concentration of $1.0 \mathrm{mg} \mathrm{mL}^{-1}$ in the precursor solution. 


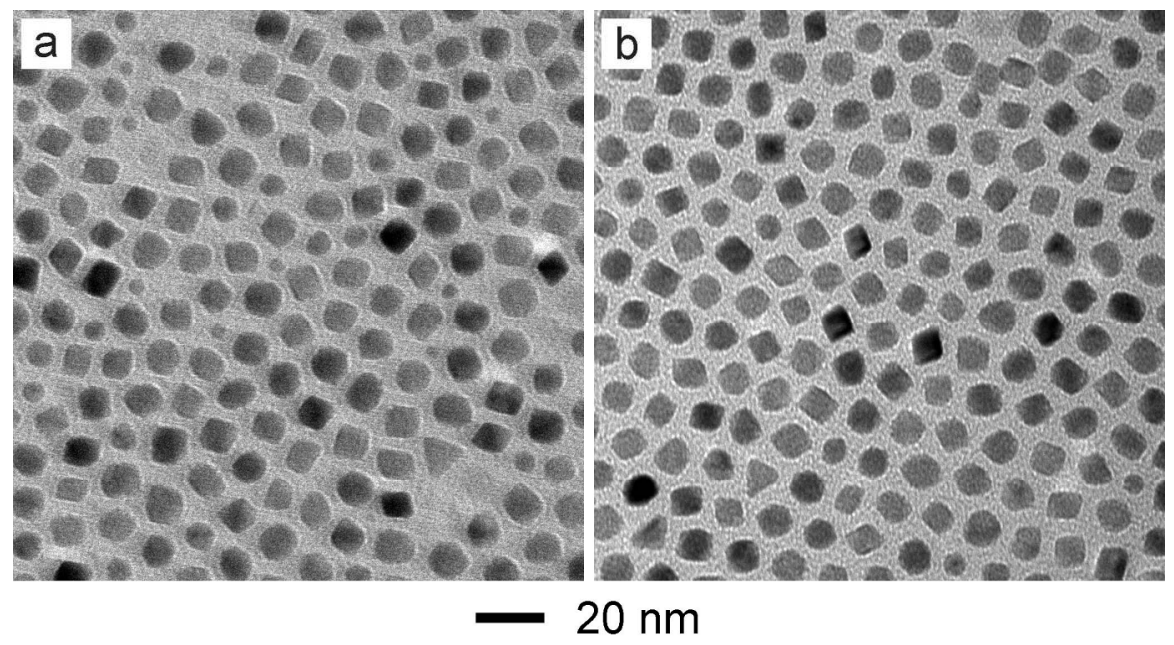

Figure S2. TEM images of the products obtained using the same protocol developed for the 9nm Pt-Ni octahedral nanocrystals, except for the different reaction temperatures: (a) 170 and (b) $200{ }^{\circ} \mathrm{C}$. 


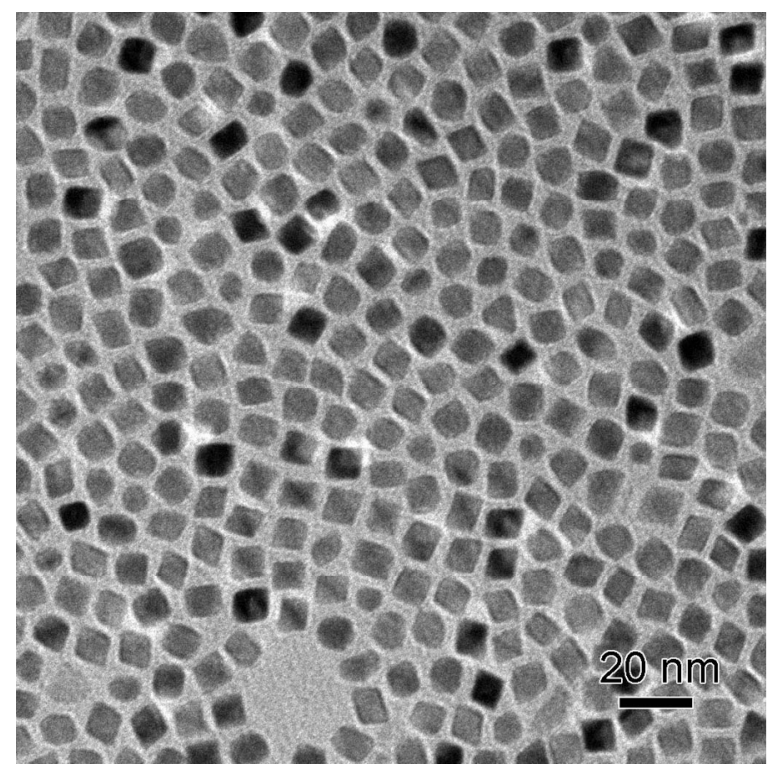

Figure S3. TEM image of the products obtained using a protocol developed for the 9-nm Pt-Ni octahedral nanocrystals, except that the PTFE tubes had an inner diameter of $3.0 \mathrm{~mm}$ and the flow rate was increased to $0.30 \mathrm{~mL} \mathrm{~min}^{-1}$. 


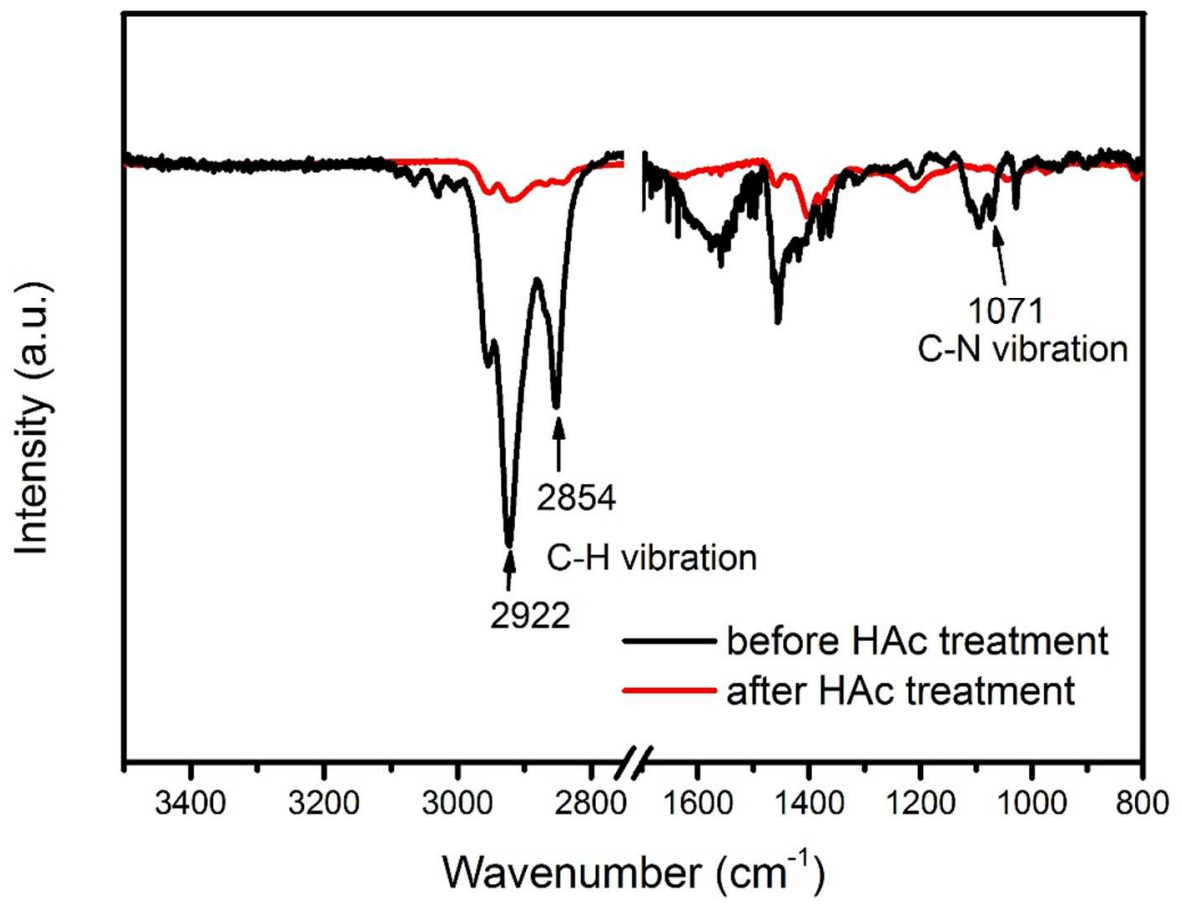

Figure S4. ATR-FTIR of the 9-nm Pt-Ni octahedral nanocrystals before (Figure 2a) and after (Figure 4a) treatment with $\mathrm{HAc}$ at $60{ }^{\circ} \mathrm{C}$ for $2 \mathrm{~h}$. Before treatment, the peaks at 2922 and 2854 $\mathrm{cm}^{-1}$ could be attributed to $\mathrm{C}-\mathrm{H}$ vibrations; the peaks at 1405 and $1305 \mathrm{~cm}^{-1}$ corresponded to the vibration modes of the $\mathrm{COO}^{-}$group in oleic acid; and the peak at $1071 \mathrm{~cm}^{-1}$ could be assigned to the $\mathrm{C}-\mathrm{N}$ vibration from oleylamine. 

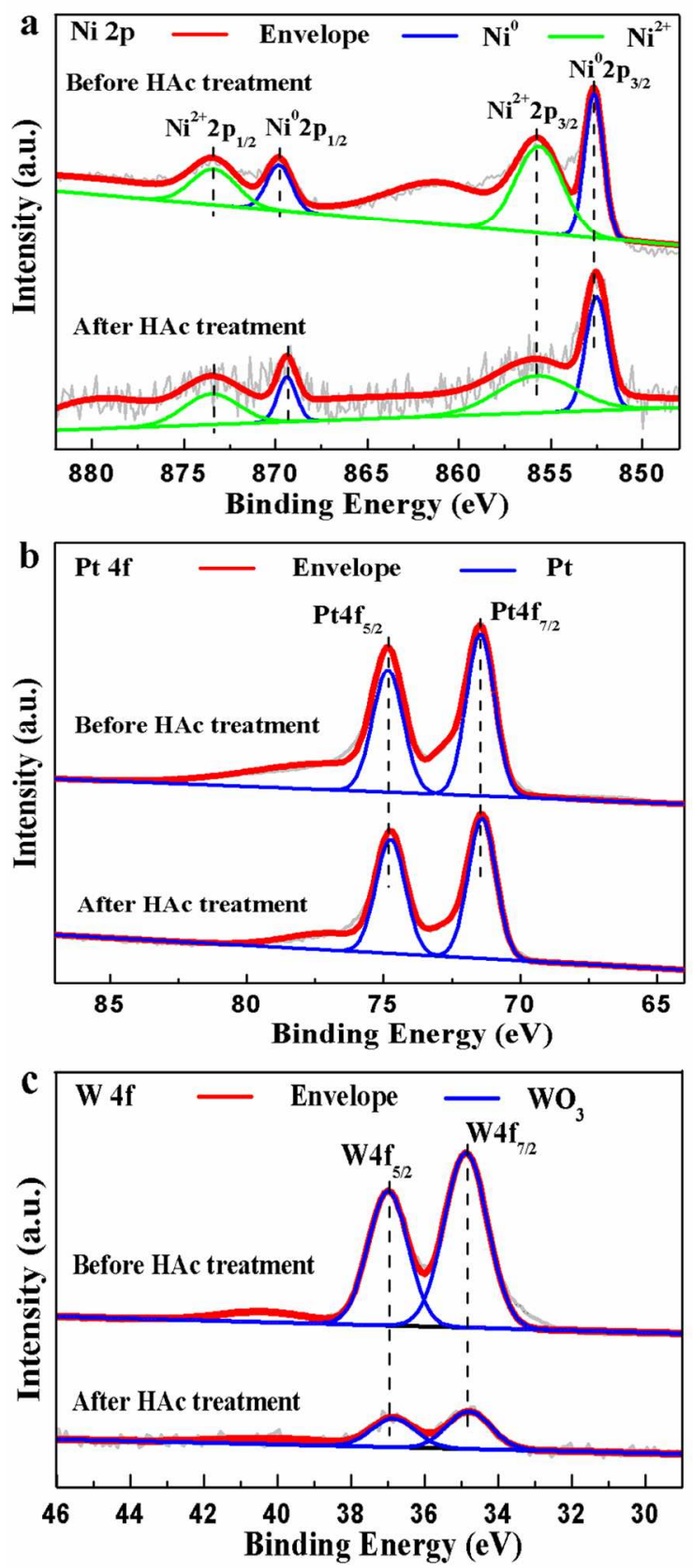

Figure S5. XPS study of the Ni (a), Pt (b), and W (c) in the 9-nm Pt-Ni octahedral nanocrystals before (Figure 2a) and after (Figure 4a) treatment with HAc at $60{ }^{\circ} \mathrm{C}$ for $2 \mathrm{~h}$. 

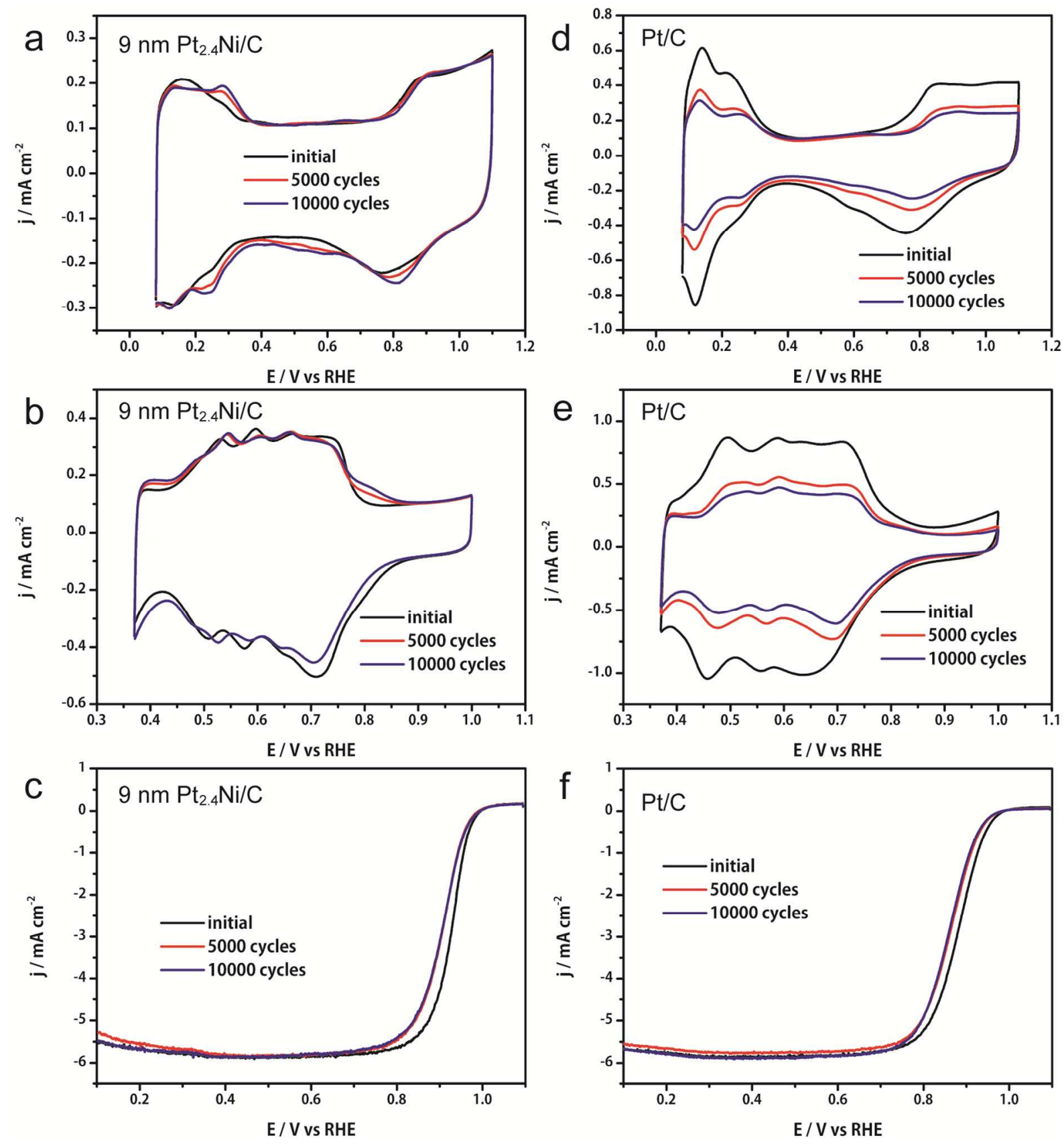

Figure S6. Accelerated durability tests of the 9-nm $\mathrm{Pt}_{2.4} \mathrm{Ni} / \mathrm{C}$ and commercial $\mathrm{Pt} / \mathrm{C}$ catalysts. (a) $\mathrm{CV}$ curves, (b) $\mathrm{Cu}_{\mathrm{UPD}}$, and (c) ORR polarization curves for the 9-nm $\mathrm{Pt}_{2.4} \mathrm{Ni} / \mathrm{C}$ before and after 5,000 and 10,000 cycles of accelerated durability tests; (d) CV curves, (e) Cu $\mathrm{u}_{\text {UPD }}$, and (f) ORR polarization curves for the $\mathrm{Pt} / \mathrm{C}$ catalyst before and after 5,000 and 10,000 cycles of accelerated durability tests. 

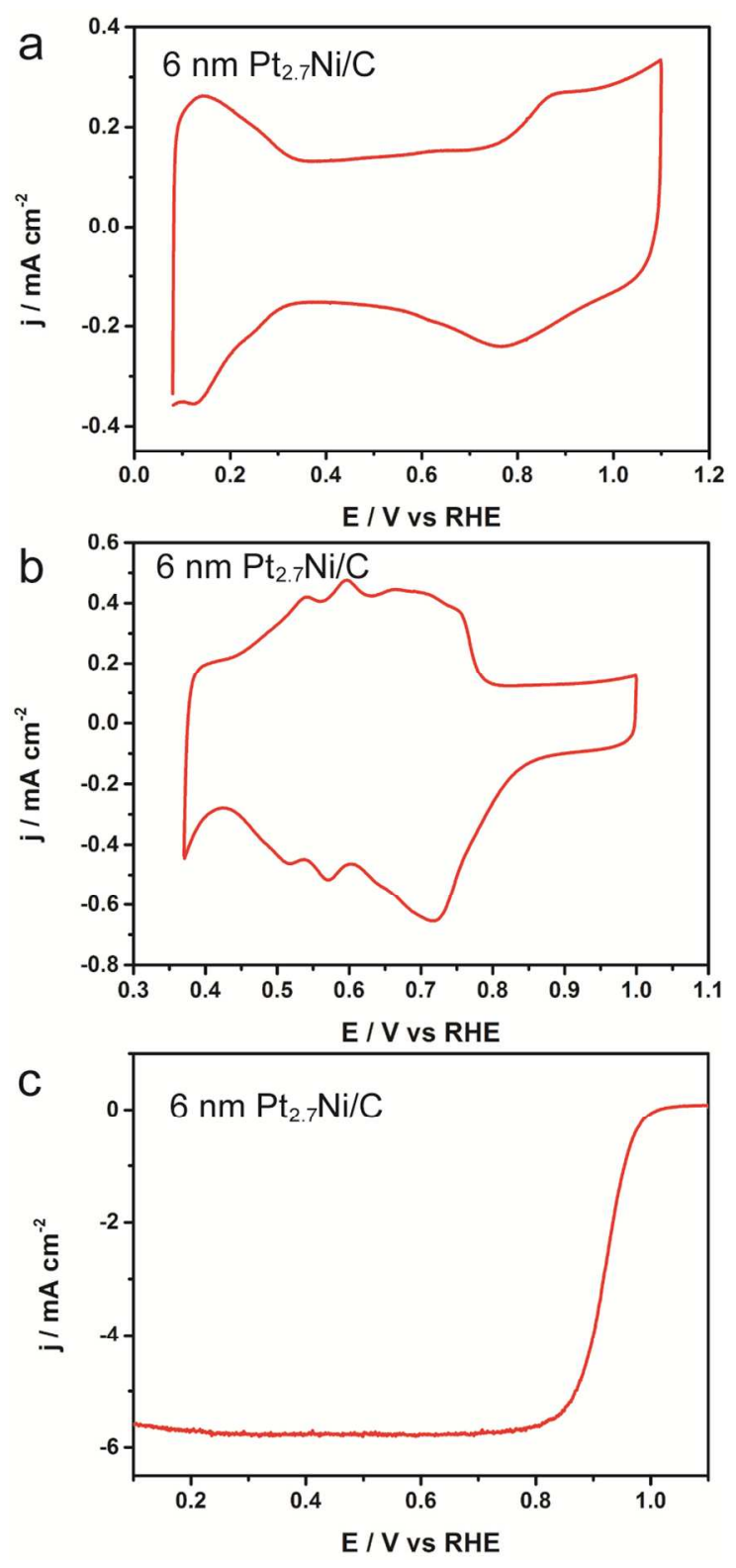

Figure S7. Electrocatalytic properties of the 6-nm $\mathrm{Pt}_{2.7} \mathrm{Ni} / \mathrm{C}$ catalyst for oxygen reduction: (a) $\mathrm{CV}$ curve; (b) $\mathrm{Cu}_{\mathrm{UPD}}$; and (c) ORR polarization curve. The ECSA calculated from $\mathrm{Cu}_{\mathrm{UPD}}$ was $52.7 \mathrm{~m}^{2} \mathrm{gpt}^{-1}$, while the specific activity at $0.9 \mathrm{~V}$ was $3.34 \mathrm{~mA} \mathrm{~cm}^{-2}$ and the mass activity was $1.76 \mathrm{~A} \mathrm{mg}_{\mathrm{Pt}}{ }^{-1}$. The loading amount of Pt on the electrode was $1.46 \mu \mathrm{g}$. 


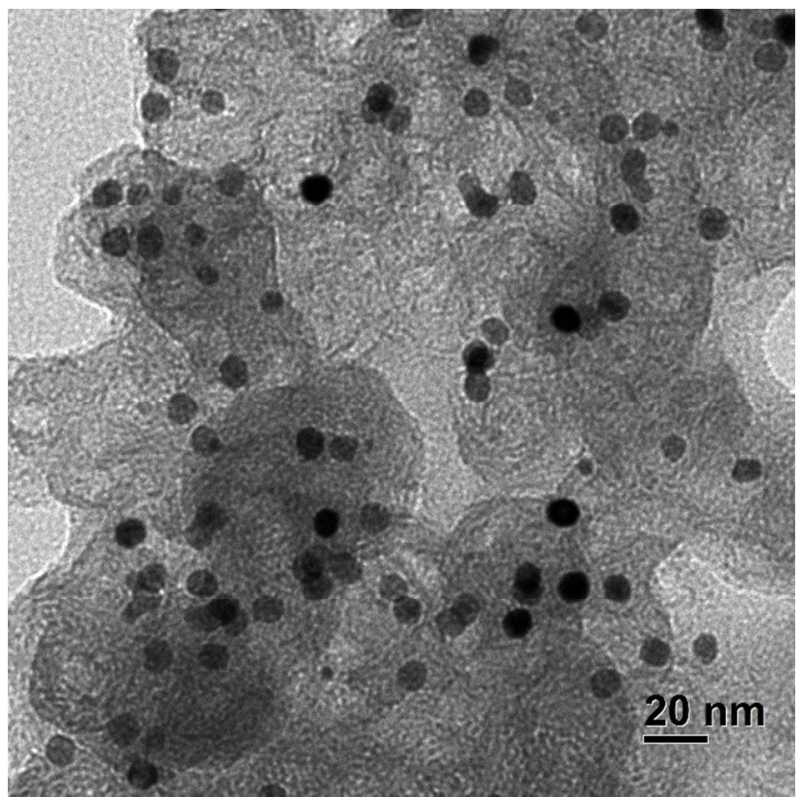

Figure S8. TEM image of the Pt-Ni nanocrystals after 10,000 cycles of accelerated durability test. The corners of the octahedral nanocrystals were rounded during the durability test and the $\mathrm{Pt} / \mathrm{Ni}$ atomic ratio changed from 2.4 to 3.4 due to the loss of $\mathrm{Ni}$. 
Table S1. The compositions of Pt-Ni octahedral nanocrystals (before and after HAc treatment) obtained at different reaction temperatures.

\begin{tabular}{|c|c|c|}
\hline \multirow{2}{*}{ Reaction temperature } & \multicolumn{2}{|c|}{ Pt/Ni atomic ratio of Pt-Ni octahedra } \\
\cline { 2 - 3 } & As-obtained & HAc treatment for $2 \mathrm{~h}$ \\
\hline $170^{\circ} \mathrm{C}$ & 1.95 & 3.41 \\
\hline $200^{\circ} \mathrm{C}$ & 1.62 & 2.78 \\
\hline $230^{\circ} \mathrm{C}$ & 1.53 & 2.40 \\
\hline
\end{tabular}

